\title{
Lung aeration and ventilation after general anesthesia in left lateral position: a prospective observational study using electrical impedance tomography
}

\author{
Yan Wang ${ }^{1 \#}$, Huisheng $\mathrm{Xu}^{1,2 \#}$, Hui Li ${ }^{1}$, Baoli Cheng ${ }^{1}$, Xiangming Fang ${ }^{1}$ \\ ${ }^{1}$ Department of Anesthesiology, The First Affiliated hospital, School of Medicine, Zhejiang University, China; ${ }^{2}$ Department of Anesthesiology, \\ Quzhou People's Hospital, Quzhou, China \\ Contributions: (I) Conception and design: Y Wang, H Xu, H Li; (II) Administrative support: None; (III) Provision of study materials or patients: \\ None; (IV) Collection and assembly of data: H Xu, B Cheng; (V) Data analysis and interpretation: Y Wang, H Xu, H Li, B Cheng; (VI) Manuscript \\ writing: All authors; (VII) Final approval of manuscript: All authors. \\ \#These authors contributed equally to this work. \\ Correspondence to: Baoli Cheng; Xiangming Fang. Department of Anesthesiology, The First Affiliated Hospital, School of Medicine, Zhejiang \\ University, Hangzhou, China. Email: chengbaoli1979@zju.edu.cn; 0099418@zju.edu.cn.
}

\begin{abstract}
Background: Endoscopic submucosal dissection (ESD) under general anesthesia in left lateral position may lead to transient impairment of pulmonary function. We used electrical impedance tomography (EIT), an increasingly implied non-invasive instrument for bedside real-time monitoring regional changes in ventilation, to assess the changes of regional lung aeration and ventilation in patients undergoing ESD.

Methods: Twenty-two patients scheduled for elective ESD under mechanical ventilation in left lateral position were studied. We acquired 2-min EIT records at four time points: (M1) baseline, before induction of anesthesia, (M2) after the start of mechanical ventilation and before surgery, (M3) after surgery and before extubation, and (M4) after extubation and before leaving operation room, respectively. To quantify regional changes in lung ventilation, we calculated the ventilation proportion of left and right lung regions. Possible changes in lung aeration were detected by changes in end-expiratory lung impedance (EELI). Global inhomogeneity index (GI) was also analyzed.
\end{abstract}

Results: After tracheal intubation in the left lateral position, left lung showed a lower ventilation proportion (M1, 49.6\% vs. M2, 36.2\% $\mathrm{P}<0.05)$, a reduction in EELI $\{\Delta \mathrm{EELI}-87$ [-809; 253]\} and a higher GI index value $(\mathrm{M} 1,0.29 \pm 0.09$ vs. $\mathrm{M} 2,0.41 \pm 0.12, \mathrm{P}<0.05)$, while right lung showed a higher ventilation proportion (M1, 50.4\% vs. M2, 63.8\%, $\mathrm{P}<0.05)$ and an increase in EELI \{ $\Delta$ EELI 161 [-952; 1,905]\}. During ESD operation, no changes in either regional ventilation distribution or GI index were observed. After extubation, the GI values in right and left lung were both returned to the level before anesthesia.

Conclusions: In patients with left lateral position undergoing ESD, left lung was characterized by decreased ventilation and more inhomogeneity while right lung was opposite after intubation. ESD procedure with carbon dioxide insufflation did not lead to significant changes in either regional ventilation or homogeneity. And the change of lung inhomogeneity during ESD procedure is transient.

Keywords: Lung ventilation; electrical impedance tomography (EIT); endoscopic submucosal dissection (ESD)

Submitted Apr 27, 2020. Accepted for publication Sep 02, 2020.

doi: 10.21037/apm-20-1029

View this article at: http://dx.doi.org/10.21037/apm-20-1029 


\section{Introduction}

Gastric cancer is globally the third leading cause of cancer death worldwide, with almost 990,000 cases of gastric cancer detected annually. Endoscopic submucosal dissection (ESD) has been regarded as an effective and minimally invasive endoscopic treatment for early gastric cancer $(1,2)$. ESD is accompanied by general anesthesia, left lateral position and repetitive carbon dioxide insufflation during the procedure. These procedural factors might create both collapse of the left part and overdistention of the right part of the lung, which may increase the risk for ventilation-induced lung injury (VILI) and possibly lead to pulmonary complications immediately thereafter $(3,4)$. Therefore, information on regional lung aeration and ventilation in patients undergoing ESD with general anesthesia is important for anesthesiologists in perioperative management.

In spontaneous breathing, the ventilation is greatest in the most dependent lung regions, which is due to the gravity gradient and to the fact that the lower diaphragm, on the dependent side, is able to contract more efficiently $(5,6)$. This is the known gravity-dependent phenomena that dependent lung regions receive a higher portion of inspired gas in lateral position during spontaneous tidal breathing. However, after induction of general anesthesia, lateral positioning has shown to cause curvature of the diaphragm by abdominal content and basal parts of the dependent lung may become atelectatic (7). In addition, the lung compliance is decreased and a distinct redistribution of ventilation in favor of the dependent regions is also happened when in lateral decubitus position (8). Traditionally, it has been suggested that gravity is the main physiological determinate that contributes to the non-homogeneous distribution of ventilation in the mechanical lungs $(9,10)$.

As a new noninvasive and radiation-free imaging tool, electrical impedance tomography (EIT) can provide instantaneous monitoring of variations in overall lung volume and regional distribution of ventilation at the bedside (11). The measuring principle of EIT is to estimate changes in intrathoracic impedance against the flow of alternating electrical currents applied to the thorax during breathing (12). EIT detects areas of reduced lung aeration by measuring a parallel reduction in electrical resistance, which reflects the fact that electrically conducting structures are less stretched and more densely arranged (13). A number of quantitative measures can be derived from EIT data to characterize ventilation distribution (14). The level of end-expiratory lung impedance (EELI) is shown to be a surrogate estimate of end-expiratory lung volume (EELV) (15). The distribution of tidal volume in the lungs of patients under mechanical ventilation is often inhomogeneous and the global inhomogeneity index (GI index) is commonly used to describe uneven air distribution within the lung $(16,17)$. As there is no radiation burden, EIT is currently the only commercially available technique that allows real-time and bedside imaging of ventilation distribution of the lungs. EIT has shown potential in optimizing ventilator settings in clinically ill patients and in reducing the risk of VILI (18-21). EIT is also especially for evaluation of therapeutic interventions such as recruitment maneuvers and PEEP titration (22-24). However, there is a lack of evidence regarding the potential application of EIT in mechanically ventilated patients with lateral position.

As patients undergoing ESD with left lateral position are prone to pulmonary complications, we prospectively investigated their regional lung aeration and ventilation changes using EIT, which may allow better monitoring of lung function. We present the following article in accordance with the STROBE reporting checklist (available at http://dx.doi.org/10.21037/apm-20-1029).

\section{Methods}

The study was conducted in accordance with the Declaration of Helsinki (as revised in 2013). This prospective observational study was approved by the local ethics committee of The First Affiliated Hospital, Zhejiang University (IIT20200029A) and registered on ClinicalTrials. org.cn (ChiCTR1900025184). This study was conducted from Aug. 2019 to Sep. 2019. Twenty-two patients undergoing elective ESD with mechanically ventilation in left lateral position were enrolled. Patients with hemodynamic instability, chronic obstructive pulmonary disease, bronchial asthma, known interstitial lung disease or any contraindication for the use of EIT such as implantable electronic cardiac devices were excluded. Written informed consents were obtained from all patients or their legal representatives.

After entering operation room, all patients were monitored with non-invasive blood pressure, peripheral oxygen saturation, and a five-lead ECG. Before anesthesia induction, patients were placed in left lateral position with a shoulder pad to ensure adequate cervical reclination and to avoid positional changes between measurements $(25,26)$. A 16-electrode EIT monitoring belt (PulmoVista 500TM EIT belt, Drägerwerk, Lübeck, Germany) was placed 
equidistantly on the thorax circumference between the 4th and 6th intercostal spaces and connected to a portable EIT monitor (PulmoVista 500TM, Drägerwerk, Lübeck, Germany) (12,27). The size of the EIT belt was chosen according to the manufacturer's recommendations. Before anesthesia induction, patients were given oxygen with mask for 5 minutes. Anesthesia was induced with midazolam 0.04 $\mathrm{mg} \cdot \mathrm{kg}^{-1}$ i.v., sufentanil $0.5 \mu \mathrm{g} \cdot \mathrm{kg}^{-1}$ i.v., propofol $2 \mathrm{mg} \cdot \mathrm{kg}^{-1}$ i.v. and vecuronium $\mathrm{mg} \cdot \mathrm{kg}^{-1}$ i.v. Following muscle relaxation, all patients were tracheally intubated and ventilated mechanically using a Primus respirator (Dräger, Lübeck, Germany) in a volume-controlled mode (tidal volume of $8 \mathrm{~mL} \cdot \mathrm{kg}^{-1}$ of ideal body weight, a standardized PEEP level of $0 \mathrm{cmH}_{2} \mathrm{O}$ and inspiration-to-expiration ratio of 1:2) (28-30). Breathing frequency was adapted to maintain end-tidal carbon dioxide partial pressure between 4.5 and $5.1 \mathrm{kPa}$. Propofol at the rate of $3-7 \mathrm{mg} \cdot \mathrm{kg}^{-1} \cdot \mathrm{h}^{-1}$, cisatracurium $2-3 \mu \mathrm{g} \cdot \mathrm{kg}^{-1} \cdot \mathrm{min}^{-1}$ and remifentanil $0.2 \mu \mathrm{g} \cdot \mathrm{kg}^{-1} \cdot \mathrm{min}^{-1}$ were continued to maintain anesthesia. And sufentanil was intermittently administered during surgery.

EIT measurements were started after a stabilization period of 5-10 minutes and obtained for 2 minutes at four predefined time points. Measurement one (M1, baseline) was carried out after arrival in the operation room in the awake, spontaneously breathing and left lateral positioning patients. During measurement two (M2), data were acquired soon after intubation and before surgery. Measurement three (M3) was made immediately after surgery and before extubation. Measurement four (M4) was performed when the patient was extubated and before leaving operation room. The horizontal images of the lungs were continuously obtained by EIT monitoring. Then the images were generated by using a modified Newton-Raphson reconstruction algorithm (Draeger EIT Data Analysis Tool 6.1; Draeger Medical GmbH). EIT reconstructed functional images with high temporal resolution based on the assessment of impedance changes during the respiratory cycle. After image reconstruction, the relative impedance changes were translated into a color scale. Impedance changes of less than $10 \%$ of the determined maximum impedance change were colored black. Impedance changes above $10 \%$ of the maximum impedance change were displayed in dark blue/light blue and white. EIT data were recorded at a scan rate of $30 \mathrm{~Hz}$, downloaded and analysed off-line (31).

From EIT, we calculated (I) regional ventilation proportion, expressed as a percentage, (II) EELI variation
( $\triangle$ EELI), values shown as arbitrary units (a.u.), (III) the inhomogeneity index, GI index, and (IV) geometrical center of ventilation, $\mathrm{CoV}$, as derived from EIT measurements. We selected the regions of interest (ROI) within the chest contours of the right and left lung based on threedimensional thoracic models. To analyze regional effects of the procedure, EIT images were primarily divided into a left ROI and a right ROI. Numeric values were continuously calculated and displayed over time to express the ventilation distribution as regional proportions. Tidal EIT images from 10 consecutive breaths were created by determining, for each pixel, the change in EELI in arbitrary units (a.u.) from baseline. GI aims to describe ventilation inhomogeneity and increased GI indicates higher heterogeneity within the set of reconstructed pixels $(17,24)$. A value close to 0.5 does not necessarily mean a homogeneous air distribution in the whole respiratory system, but a value close to 1 definitely means inhomogeneous distribution between the left and right lungs according to EIT data. Based on the differential images obtained between different time points, a series of GI indices were calculated as previous reports (17). The $\mathrm{CoV}$ was a previously described parameter to express the geometrical center of tidal distribution within the thoracic cross section (32).

\section{Statistical analysis}

An a priori power calculation $\left(G^{*}\right.$ Power 3.1.9.2, Universities of Kiel and Dusseldorf, Germany) revealed a sample size of 19 to be sufficient to detect a difference in lung ventilation with calculated effect size of 0.28 to achieve an error probability for $\alpha=0.05$ and $1-\beta=0.80$. Data analysis was performed using GraphPad Prism 6.0 (GraphPad Software, La Jolla, CA, USA). Data were tested for normal distribution with the Kolmogorov-Smirnov normality test. Quantitative variables are presented as mean and standard deviation (SD) or median and interquartile range. Qualitative variables are expressed as proportions. Data were tested using $t$-tests or $\mathrm{U}$ tests, as appropriate. For changes of parameters between the time points, repeated measures analyses of variance (ANOVA) were used. A P value $<0.05$ was considered statistically significant.

\section{Results}

A total of 22 consecutive patients were included in this study. Baseline parameters presenting the physiologic and 
Table 1 Baseline characteristics of patients $(n=22)$

\begin{tabular}{ll}
\hline Characteristics & Outcome \\
\hline Age, years & $61.3 \pm 1.3$ \\
Females/males & $7 / 15$ \\
$\mathrm{BMI}, \mathrm{kg} / \mathrm{m}^{2}$ & $21.5 \pm 1.5$ \\
$\mathrm{ASAl} / \mathrm{Il}$ & $13 / 9$ \\
Surgery duration, minutes & $71.9 \pm 8.7$ \\
Duration between after surgery and leaving operation & $18.9 \pm 1.5$ \\
room, minutes & \\
Length of hospital stay, days & $4.6 \pm 0.3$ \\
\hline BMl, body mass index; ASA, American Society of Anesthesiology.
\end{tabular}

demographic data of the study population were summarized in Table 1.

\section{Regional ventilation proportion}

Examples of EIT images from one representative patient at predefined four time points were depicted in Figure1. Comparison of right lung ventilation at the four distinct time points of the procedure showed significant increase after intubation $(\mathrm{M} 1,50.4 \%$ vs. $\mathrm{M} 2,63.8 \%, \mathrm{P}<0.05)$ and then returned to the baseline level after extubation (M1, $50.4 \%$ vs. M4, 59.4\%, $\mathrm{P}>0.05)$. While, comparison of left lung ventilation at the four distinct time points of the procedure showed significant decrease after intubation (M1, $49.6 \%$ vs. $\mathrm{M} 2,36.2 \%, \mathrm{P}<0.05)$ and then returned to the baseline level after extubation (M1, 49.6\% vs. M4, 40.6\%, $\mathrm{P}>0.05)$. During ESD operation, no changes in ventilation proportion of right lung or left lung were observed between M2 and M3 (Figure 2A,B).

The assessment of the lungs with the use of EIT showed ventilated areas were similar in the right and left lungs at baseline (50.4\% vs. $49.6 \%)$. There was increased ventilation proportion of right lung compared with left lung after intubation and after surgery and before extubation, respectively $(63.8 \%$ vs. $36.2 \%$, $64.0 \%$ vs. $36.3 \%$, both $\mathrm{P}<0.001)$. After extubation, the ventilation proportion of right lung was still higher than that of left lung $(59.4 \% \mathrm{vs}$. 40.6\%) (Figure 2C,D,E,F).

\section{$\triangle E E L I$}

After intubation in left lateral position, an increase in EELI was seen within the right aspect of the measured thoracic cross section, while a decrease with the left ROI was observed \{ $\triangle$ EELI right 161 [-952; 1,905]; left -87 [-809; 253]\}. EIT measurements following extubation also revealed regional effects of this procedure: The right and left lung regions showed a significant decrease in aeration compared with the level after surgery and before extubation $\{\triangle \mathrm{EELI}$ right -497 [-960; -101]; left -268 [-2,294; 143]\} (Table 2).

\section{GI index}

Figure 3 presents a comparison of the GI index values at four time points. The mean GI value of each patient was used for comparison. After intubation, the GI index increased in total (M1, $0.37 \pm 0.09$ vs. $\mathrm{M} 2,0.45 \pm 0.07, \mathrm{P}<0.05)$ and left lung (M1, $0.29 \pm 0.09$ vs. $M 2,0.41 \pm 0.12, \mathrm{P}<0.05)$. After surgery and before extubation, it did not significantly differ from the GI value before surgery and after intubation in total $(\mathrm{M} 2,0.45 \pm 0.07$ vs. M3, $0.44 \pm 0.06)$ and left lung (M2, $0.41 \pm 0.12$ vs. M3, $0.37 \pm 0.10$ ). A significantly higher GI value was found compared with baseline in total (M1, $0.37 \pm 0.09$ vs. $\mathrm{M} 3,0.44 \pm 0.06, \mathrm{P}<0.05)$ and left lung (M1, $0.29 \pm 0.09$ vs. $\mathrm{M} 3,0.37 \pm 0.10, \mathrm{P}<0.05)$. After extubation, the GI value was decreased to the level at baseline. In right lung, significant differences were found in GI values after surgery and before extubation when compared to baseline (M1, $0.35 \pm 0.078 v s$. M3, $0.39 \pm 0.07, \mathrm{P}<0.05)$. After extubation, the GI index was returned to the level that was similar with the baseline.

\section{Center of ventilation measurement}

Accordingly, $\mathrm{CoV}$ did not show significant changes over the course of time points from M1 to M4 (M1, 49.8\% vs. M2, 47.1\%, vs. M3, 47.4\% vs. M4, 49.6\%) (Figure 4).

\section{Clinical outcomes}

Summary of clinical outcomes is shown in Table 3. No patient was detected with postoperative hypoxemia, cough, pulmonary edema, pneumonia or atelectasis. Two patients (9\%) had fever after surgery. In addition, no patient was treated with atomization inhalation or transferred to intensive care unit postoperatively.

\section{Discussion}

In this study aimed at assessing, in mechanically ventilated patients with left lateral position, changes in reginal lung 
A
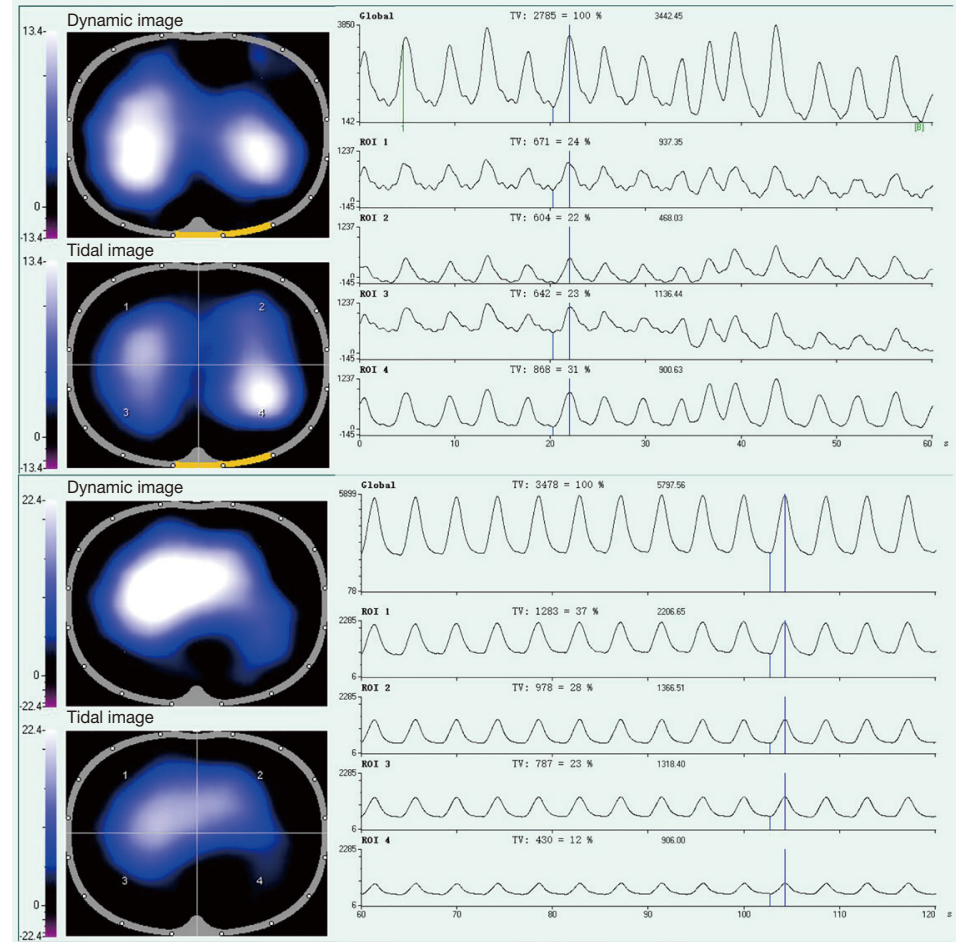

B

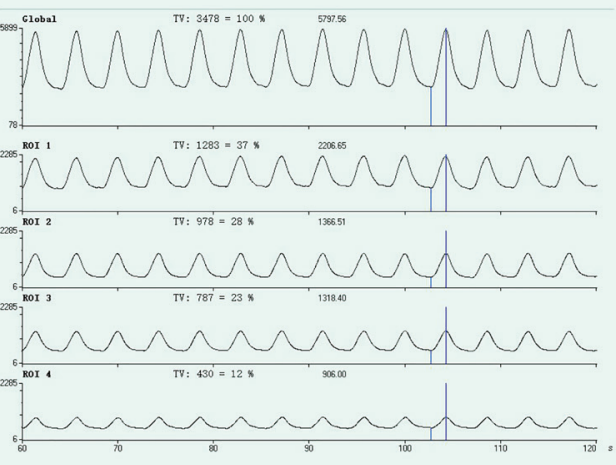

C
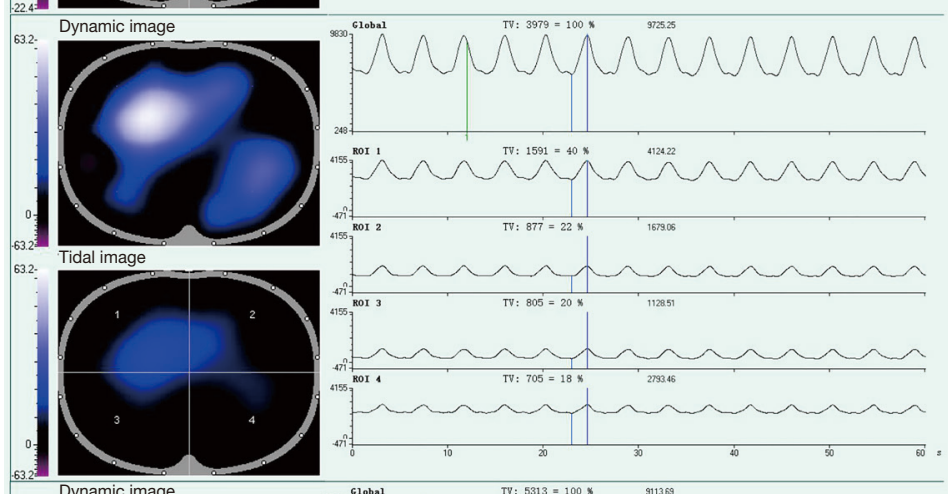

$\mathrm{D}$
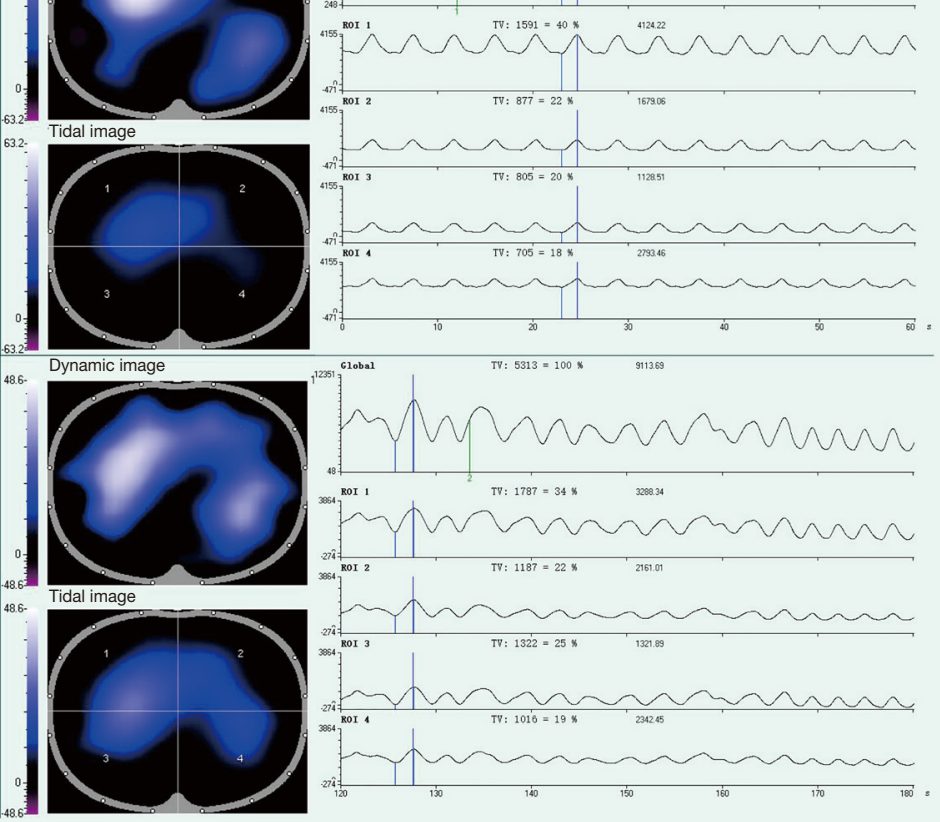

Figure 1 Ventilation map reconstructed by EIT from one representative patient at predefined four time points using a color scale: the lighter the blue, the greater the regional ventilation. Dynamic image represents the changes in tissue properties between a baseline (reference) measurement frame and the current frame. Tidal image represents the difference of impedance between end-inspiration and end-expiration. (A) Regional ventilation at M1; (B) regional ventilation at M2; (C) regional ventilation at M3; (D) regional ventilation at M4. M1, after arrival in the operation room; M2, soon after intubation and before surgery; M3, immediately after surgery and before extubation; M4, after extubated and before leaving operation room; ROI, region of interest; TV, tidal volume. 

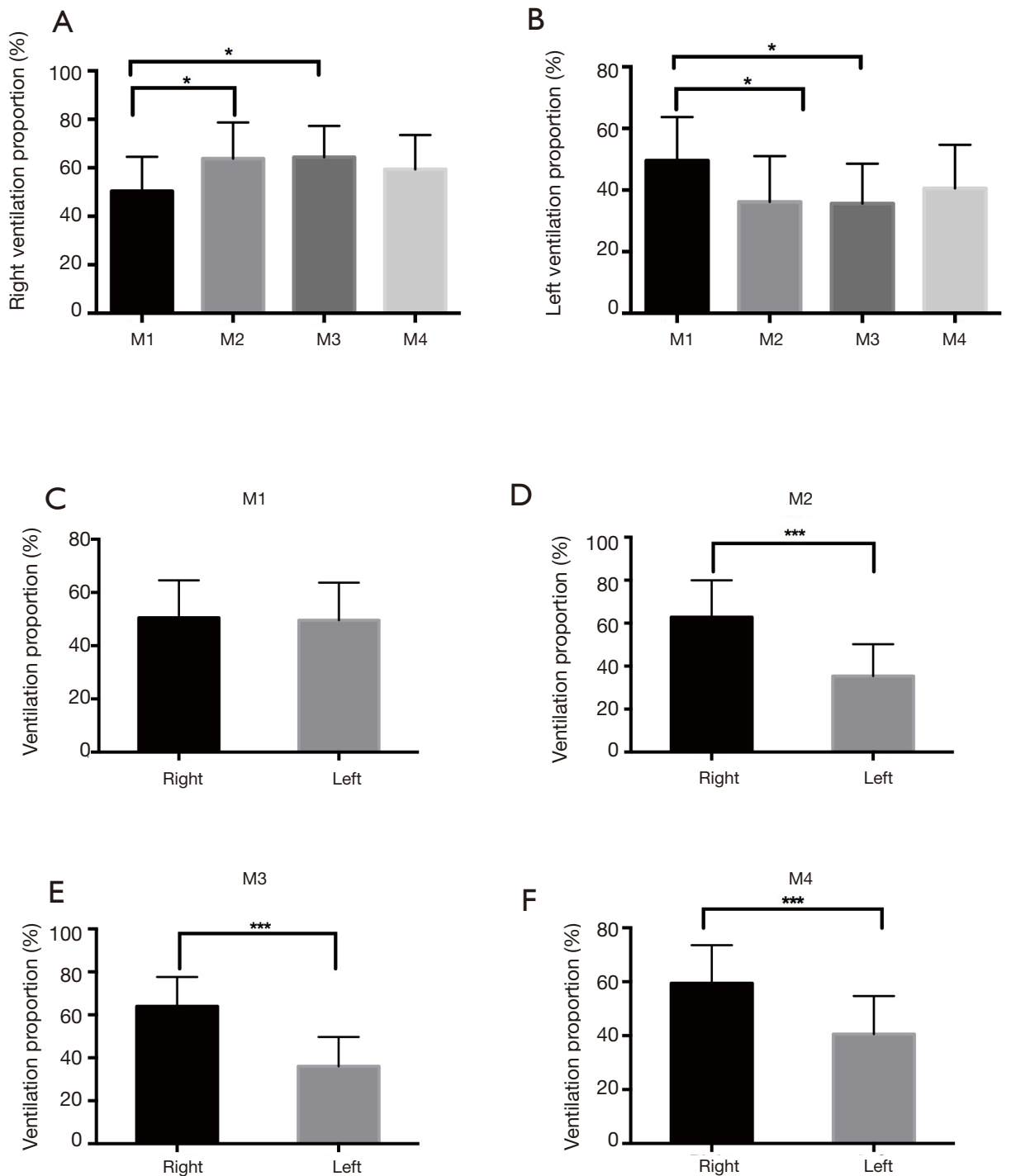

Figure 2 Ventilation proportion of right lung and left lung using EIT. (A) Ventilation proportion of right lung at different time point; (B) ventilation proportion of left lung at different time points; (C) comparison of ventilation proportion between right and left lung at M1; (D) comparison of ventilation proportion between right and left lung at M2; (E) comparison of ventilation proportion between right and left lung at M3 and (F) comparison of ventilation proportion between right and left lung at M4. M1, after arrival in the operation room; M2, soon after intubation and before surgery; M3, immediately after surgery and before extubation; M4, after extubated and before leaving operation room; *, significant difference, $\mathrm{P}<0.05$; ${ }^{* * *}$, significant difference, $\mathrm{P}<0.001$.

Table 2 Changes in EELI

\begin{tabular}{lll}
\hline Variable & Right lung & Left lung \\
\hline$\Delta$ EELI measured: after intubation & $161[-952 ; 1,905]$ & $-87[-809 ; 253]$ \\
$\Delta$ EELI measured: after extubation & $-497[-960 ;-101]$ & $-268[-2,294 ; 143]$ \\
\hline
\end{tabular}



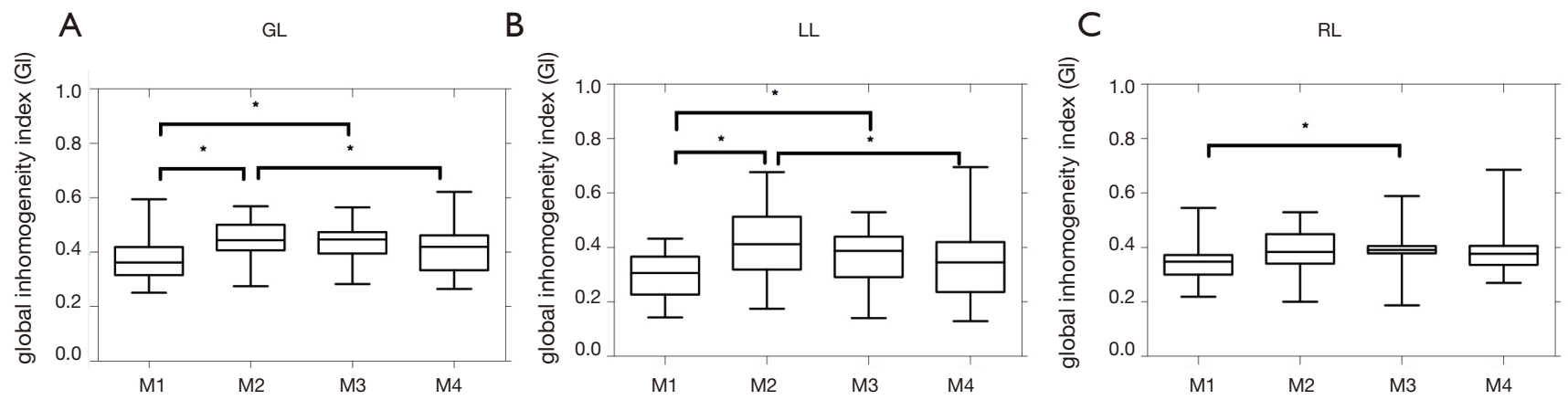

Figure 3 Global inhomogeneity index measured by EIT. (GL), global inhomogeneity index at different time points in global lung; (LL), global inhomogeneity index at different time points in left lung; (RL), global inhomogeneity index at different time points in right lung. M1, after arrival in the operation room; M2, soon after intubation and before surgery; M3, immediately after surgery and before extubation; M4, after extubated and before leaving operation room; GL, global lung; RL, right lung; LL, left lung; GI, global inhomogeneity; *, significant difference, $\mathrm{P}<0.05$.

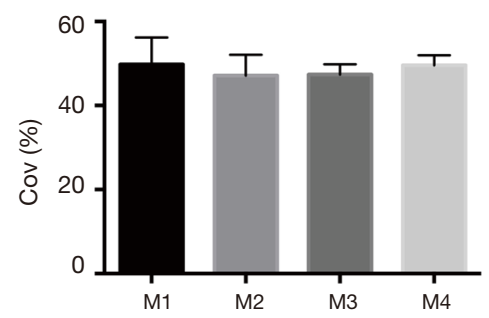

Figure 4 Center of ventilation measurement using EIT at different time points. M1, after arrival in the operation room; M2, soon after intubation and before surgery; $M 3$, immediately after surgery and before extubation; M4, after extubated and before leaving operation room; $\mathrm{CoV}$, center of ventilation.

Table 3 Clinical outcomes for 22 patients undergoing ESD in left lateral position

\begin{tabular}{lll}
\hline Variable & Number & Percentage \\
\hline Hypoxemia & 0 & 0 \\
Fever & 2 & $9 \%$ \\
Cough & 0 & 0 \\
Pulmonary edema & 0 & 0 \\
Pneumonia & 0 & 0 \\
Atelectasis & 0 & 0 \\
Atomization inhalation & 0 & 0 \\
treatment & & 0 \\
Transfer to intensive care unit & 0 & \\
\hline
\end{tabular}

aeration, ventilation distribution and inhomogeneity occurring during ESD process, we found the following: (I) after intubation, left lung was characterized by decreased ventilation, as suggested by the lower ventilation proportion, reduction in EELI reflecting in a fall in EELV and greater lung inhomogeneity, as indicated by higher GI index value, while right lung was opposite as higher ventilation proportion and increase in EELI; (II) ESD operation with carbon dioxide insufflation did not lead to significant changes in either regional ventilation distribution or homogeneity; and (III) after extubation, regional lung homogeneity was back to the level before anesthesia. Taken together, these findings suggest temporary changes of lung status during the whole ESD surgery.

As the application of minimally invasive surgery including ESD has increased over the years, bedside tools capable of identifying the consequences of therapeutic interventions with sufficient sensitivity and specificity could allow optimization of ventilation strategies for such settings. Recent studies have shown that the concept of lungprotective ventilation might have a positive impact not only in critically ill patients, but also in patients without known lung injury who are undergoing surgery $(33,34)$. During ESD under general anesthesia in left lateral position, gas exchange is impaired because of a mismatch of the regional distribution of ventilation and perfusion (35). The main pathogenic mechanism is the development of atelectasis in left lung areas and over distension in right lung areas (36). 
Given that surgery procedure and artificial ventilation in lateral position can cause harm to healthy lungs, detecting regional ventilation to guide strategies for such patients have been especially advocated. However, identification of regional lung dysfunction and monitoring of surgery effects at the bedside still remains difficult, because global parameters of lung mechanics provide only integral information, lacking resolution at the regional level. EIT could provide regional information on pulmonary status of patients. Further, the gravity-related topographical changes of intrapulmonary gas distribution in the lungs associated with lateral position could be established by EIT. Therefore, in this study we use EIT continuous imaging to help assess the changes of regional lung aeration and ventilation in patients with left lateral position undergoing ESD.

Numerous studies examined the application of EIT on mechanically ventilated patients in the supine position. As we could show in the present study, EIT has the unique ability to display regional ventilation defects in lateral position right at the bedside. As the main result of study, we found that in spontaneously breathing patients with left lateral position, ventilated areas were similar in the left and right lungs. While other studies indicate the finding of greater ventilation in the dependent lung in spontaneous breathing (5). This consistency may be due to the effect of placing the electrodes in transverse planes at different thoracic levels. With the induction of anesthesia, ventilation in the right lung regions increased significantly while in the left lung regions decreased significantly, suggesting a ventilation-perfusion mismatch. Moreover, analysis of ventilation proportion of left and right lung indicated significant difference after intubation until to the end of surgery. This finding corresponds with previous studies which showed a significant shift of ventilation towards the nondependent lung after being positioned on their side (7). In left lateral position, the ventilation gradient is redistributed along the gravitational axis. The subsequent compression of left lung areas by right abdominal organs may be the most important pathophysiological causes of "left lateral positioning anesthesia-induced atelectasis" (37). Moreover, during surgery, there was not significantly more ventilation in the right lung or less ventilation in the left lung happened, indicating carbon dioxide insufflation during ESD procedure had no significant effect on regional ventilation.

This study also showed that ESD under general anesthesia with left lateral position led to regionally dissimilar alterations of EELI. As the effects of positioning changes of patients on the EELI were described, patients in our study were remained in left positioning between four measurements and we thoroughly avoided any positioning alteration (38). A fall in EELI may just reflect aeration loss. The observed reduction in EELI in left lung areas after intubation is suggestive of collapse caused by left lateral position. An increase in EELI within the right aspect of the scan volume indicates increased regional aeration after intubation. The observed changes in EELI distribution most likely result from the same gravitational factors that contribute to the non-homogeneous aeration distribution in patients with left lateral $(9,10)$. The results were consistent with previous studies that also suggest the ventilation-related changes in impedance along the craniocaudal axis were in agreement with traditional respiratory physiology (37). Based on the results of the present study, the EELI derived by EIT could in future serve as such non-invasive and continuously available parameters.

Ventilation inhomogeneity is associated with overall disease severity and mortality, an intervention that decreases ventilation inhomogeneity could therefore, theoretically, result in improved patients outcomes $(39,40)$. EIT is commonly used to identify heterogeneities in the distribution of ventilation caused by pathologies and ventilator settings. The GI, as one compact index, is able to describe the inhomogeneity of tidal volume distribution in the entire lung (24). In the present study, the common inhomogeneity measure GI was used to quantify the lung condition in patients undergoing ESD with left lateral position. In general, we found a similar trend in total lung and left lung that GI index appears to be greater after intubation. Under condition of anesthesia in left lateral position, the alveoli in the left lung may collapse, which may lead to an inhomogeneous air distribution in the entire lung $(41,42)$. In addition, we failed to observe differences in the GI index during operation period, indicating ESD operation with carbon dioxide insufflation did not cause changes with respect to ventilation inhomogeneity. After extubation, ventilation distribution became more homogenous, as indicated by the decrease of the GI index that equals to the level at baseline in the entire lung, suggesting temporary changes of lung inhomogeneity during the whole ESD surgery. The EIT-based GI index could potentially help clinicians to optimize mechanical ventilation during general anaesthesia in left lateral position by the adjustment of 
PEEP, tidal volumes, and recruitment manoeuvres in the future. This interplay of personalized ventilation parameters to mitigate the side effects of anesthesia and surgery may achieve the best possible protective effect for the lungs.

The reliability of EIT results has already been confirmed by several clinical and animal studies (43-45). Although the resolution of the images is limited, EIT can be accurately visualized in the regional ventilation, which makes it an ideal candidate for early detection of atelectasis (46). Moreover, spatial resolution of EIT may not be sufficient to detect minor changes as it has been shown to be inferior to computed tomography scans, which are considered as gold standard of lung imaging. This means formation of subtle atelectases may have been missed in our investigation.

Our study has certain limitations. We did not compare different EIT-guided ventilation settings but merely documented how ventilation conditions and body positions affected the function of the lungs. We also did not measure lung perfusion using EIT, as matching on V/ $\mathrm{Q}$ is also important for final gas exchange. In addition, the oxygenation of the studied patients was lack, and it would be of interest how lung aeration changed with changes in oxygenation during ESD. We excluded patients with preexisting pulmonary diseases. However, further investigations in these patients to get benefit from EIT by the application of a targeted ventilatory approach are inevitable.

\section{Conclusions}

In patients with left lateral position undergoing ESD, left lung was characterized by decreased ventilation and more inhomogeneity while right lung was opposite after intubation. ESD procedure did not lead to significant changes in either regional ventilation distribution or homogeneity. After extubation, regional homogeneity returned to the level before anesthesia, supporting the notion that the effect of ESD operation with carbon dioxide insufflation and general anesthesia in left lateral positioning on lung condition is transient. Therefore, EIT may be a promising clinical tool for continuous and noninvasive monitoring of pulmonary status that can be especially useful in mechanically ventilated patients with left lateral position during surgery. More clinical validation studies are awaited to explore the full potential of the technology.

\section{Acknowledgments}

Funding: This work was supported by Ministry of Science and Technology of China (2018YFC2001903 to Hui Li and 2018YFC2001904 to Xiangming Fang).

\section{Footnote}

Reporting Checklist: The authors have completed the STROBE reporting checklist. Available at http://dx.doi. org/10.21037/apm-20-1029

Data Sharing Statement: Available at http://dx.doi. org/10.21037/apm-20-1029

Peer Review File: Available at http://dx.doi.org/10.21037/ apm-20-1029

Conflicts of Interest: All authors have completed the ICMJE uniform disclosure form (available at http://dx.doi. org/10.21037/apm-20-1029). The authors have no conflicts of interest to declare.

Ethical Statement: The authors are accountable for all aspects of the work in ensuring that questions related to the accuracy or integrity of any part of the work are appropriately investigated and resolved. The study was conducted in accordance with the Declaration of Helsinki (as revised in 2013). This prospective observational study was approved by the local ethics committee of The First Affiliated Hospital, Zhejiang University and registered on ClinicalTrials.org.cn (ChiCTR1900025184). Written informed consents were obtained from all patients or their legal representatives.

Open Access Statement: This is an Open Access article distributed in accordance with the Creative Commons Attribution-NonCommercial-NoDerivs 4.0 International License (CC BY-NC-ND 4.0), which permits the noncommercial replication and distribution of the article with the strict proviso that no changes or edits are made and the original work is properly cited (including links to both the formal publication through the relevant DOI and the license). See: https://creativecommons.org/licenses/by-nc-nd/4.0/.

\section{References}

1. Fujishiro M, Yoshida S, Matsuda R, et al. Updated evidence on endoscopic resection of early gastric cancer from Japan. Gastric Cancer 2017;20:39-44.

2. Gu L, Khadaroo PA, Chen L, et al. Comparison of Long- 
Term Outcomes of Endoscopic Submucosal Dissection and Surgery for Early Gastric Cancer: a Systematic Review and Meta-analysis. J Gastrointest Surg 2019;23:1493-501.

3. Takada J, Araki H, Mizutani T, et al. Safety of Carbon Dioxide Insufflation during Endoscopic Submucosal Dissection for Esophageal Squamous Cell Carcinoma. Dig Dis 2019;37:93-9.

4. Matsumi A, Takenaka R, Ando C, et al. Preoperative Pulmonary Function Tests Predict Aspiration Pneumonia After Gastric Endoscopic Submucosal Dissection. Dig Dis Sci 2017;62:3084-90.

5. Kao TJ, Amm B, Wang X, et al. Real time 3D electrical impedance imaging for ventilation and perfusion of the lung in lateral decubitus position. Annu Int Conf IEEE Eng Med Biol Soc 2014;2014:1135-8.

6. Tobin MJ. Principles and practice of mechanical ventilation. Third Edition, McGraw-Hill, 2012:306.

7. Šribar A, Merc V, Persec Z, et al. Influence of different PEEP levels on electrical impedance tomography findings in patients under general anesthesia ventilated in the lateral decubitus position. J Clin Monit Comput 2020;34:311-8.

8. Thomas PJ, Paratz JD, Lipman J, et al. Lateral positioning of ventilated intensive care patients: a study of oxygenation, respiratory mechanics, hemodynamics, and adverse events. Heart Lung 2007;36:277-86.

9. Glenny RW. Determinants of regional ventilation and blood flow in the lung. Intensive Care Med 2009;35:1833-42.

10. West JB. Regional differences in the lung. Chest 1978;74:426-37.

11. Trepte CJ, Phillips CR, Sola J, et al. Electrical impedance tomography (EIT) for quantification of pulmonary edema in acute lung injury. Crit Care 2016;20:18.

12. Bikker IG, Preis C, Egal M, et al. Electrical impedance tomography measured at two thoracic levels can visualize the ventilation distribution changes at the bedside during a decremental positive end-expiratory lung pressure trial. Crit Care 2011;15:R193.

13. Bodenstein M, David M, Markstaller K. Principles of electrical impedance tomography and its clinical application. Crit Care Med 2009; 37:713-24.

14. Frerichs I, Amato MB, van Kaam AH, et al. Chest electrical impedance tomography examination, data analysis, terminology, clinical use and recommendations: consensus statement of the TRanslational EIT developmeNt stuDy group. Thorax 2017;72:83-93.

15. Spinelli E, Mauri T, Fogagnolo A, et al. Electircal impedance tomography in periopertative medicine: careful respiratory monitoring for tailored interventions. BMC Anesthesiol 2019;19:140.

16. Lobo B, Hermosa C, Abella A, et al. Electrical impedance tomography. Ann Transl Med 2018;6:26.

17. Zhao Z, Moller K, Steinmann D, et al. Evaluation of an electrical impedance tomography-based Global Inhomogeneity Index for pulmonary ventilation distribution. Intensive Care Med 2009;35:1900-6.

18. Pulletz S, Kott M, Elke G, et al. Dynamics of regional lung aeration determined by electrical impedance tomography in patients with acute respiratory distress syndrome. Multidiscip Respir Med 2012;7:44.

19. Bellani G, Mauri T, Pesenti A. Imaging in acute lung injury and acute respiratory distress syndrome. Curr Opin Crit Care 2012;18:29-34.

20. Bauer M, Opitz A, Filser J, et al. Perioperative redistribution of regional ventilation and pulmonary function: a prospective observational study in two cohorts of patients at risk for postoperative pulmonary complications. BMC Anesthesiol 2019;19:132.

21. Bachmann MC, Morais C, Bugedo G, et al. Electrical impedance tomography in acute respiratory distress syndrome. Crit Care 2018;22:263.

22. Cinnella G, Grasso S, Raimondo P, et al. Physiological Effects of the Open Lung Approach in Patients with Early, Mild, Diffuse Acute Respiratory Distress Syndrome: An Electrical Impedance Tomography Study. Anesthesiology 2015;123:1113-21.

23. Liu K, Huang C, Xu M, et al. PEEP guided by electrical impedance tomography during one-lung ventilation in elderly patients undergoing thoracoscopic surgery. Ann Transl Med 2019;7:757.

24. Zhao Z, Pulletz S, Frerichs I, et al. The EIT-based global inhomogeneity index is highly correlated with regional lung opening in patients with acute respiratory distress syndrome. BMC Res Notes 2014;7:82.

25. Li H, Wang W, Lu YP, et al. Evaluation of endotracheal intubation with a flexible fiberoptic bronchoscope in lateral patient positioning: a prospective randomized controlled trial. Chin Med J (Engl) 2016;129:2045-9.

26. Jin Y, Ying J, Zhang K, et al. Endotracheal intubation under video laryngoscopic guidance during upper gastrointestinal endoscopic surgery in the left lateral position: a randomized controlled trial. Medicine (Baltimore) 2017;96:e9461.

27. Bialka S, Copik M, Rybczyk K, et al. Assessment of changes of regional ventilation distribution in the lung tissue depending on the driving pressure applied 
during high frequency jet ventilation. BMC Anesthesiol 2018;18:101.

28. Young CC, Harris EM, Vacchiano C, et al. Lungprotective ventilation for the surgical patient: international expert panel-based consensus recommendations. Br J Anaesth 2019;123:898-913.

29. Yang D, Grant MC, Stone A, et al. A meta-analysis of intraoperative ventilation strategies to prevent pulmonary complicaitons: is low tidal volume alone sufficient to protect healthy lungs? Ann Surg 2016;263:881e7.

30. Cai H, Gong H, Zhang L, et al. Effect of low tidal volume ventilation on atelectasis in patients during general anesthesia: a computed tomographic scan. J Clin Anesth 2007;19:125e9.

31. Longhini F, Maugeri J, Andreoni C, et al. Electrical impedance tomography during spontaneous breathing trials and after extubation in critically ill patients at high risk for extubation failure: a multicenter observational study. Ann Intensive Care 2019;9:88.

32. Radke OC, Schneider T, Heller AR, et al. Spontaneous breathing during general anesthesia prevents the ventral redistribution of ventilation as detected by electrical impedance tomography: a randomized trial. Anesthesiology 2012;116:1227-34.

33. Determann RM, Royakkers A, Wolthuis EK, et al. Ventilation with lower tidal volumes as compared with conventional tidal volumes for patients without acute lung injury: a preventive randomized controlled trial. Crit Care 2010;14:R1.

34. Severgnini P, Selmo G, Lanza C, et al. Protective mechanical ventilation during general anesthesia for open abdominal surgery improves postoperative pulmonary function. Anesthesiology 2013;118:1307-21.

35. Kim MJ, Hwang HJ, Song HH. A randomized trial on the effects of body positions on lung function with acute respiratory failure patients. Int J Nurs Stud 2002;39:549-55.

36. Rothen HU, Sporre B, Engberg G, et al. Airway closure,

Cite this article as: Wang $\mathrm{Y}, \mathrm{Xu} \mathrm{H}, \mathrm{Li} \mathrm{H}$, Cheng B, Fang $X$. Lung aeration and ventilation after general anesthesia in left lateral position: a prospective observational study using electrical impedance tomography. Ann Palliat Med 2021;10(2):1285-1295. doi: 10.21037/apm-20-1029 atelectasis and gas exchange during general anaesthesia. $\mathrm{Br}$ J Anaesth 1998;81:681-6.

37. Ericsson E, Tesselaar E, Sjoberg F. Effect of electrode belt and body positions on regional pulmonary ventilation and perfusion related impedance changes measured by electric impedance tomography. PLoS One 2016;11:e0155913.

38. Vogt B, Mendes L, Chouvarda I, et al. Influence of torso and arm positions on chest examinations by electrical impedance tomography. Physiol Meas 2016;37:904-21.

39. Cressoni M, Cadringher P, Chiurazzi C, et al. Lung inhomogeneity in patients with acute respiratory distress syndrome. Am J Respir Crit Care Med 2014;189:149-58.

40. Becher T, Vogt B, Kott M, et al. Functional Regions of Interest in Electrical Impedance Tomography: A Secondary Analysis of Two Clinical Studies. PLoS One 2016;11:e0152267.

41. Tusman G, Bohm SH, Suarez-Sipmann F, et al. Alveolar recruitment improves ventilatory efficiency of the lungs during anesthesia. Can J Anaesth 2004;51:723-7.

42. Hedenstierna G, Edmark L. The effects of anesthesia and muscle paralysis on the respiratory system. Intensive Care Med 2005;31:1327-35.

43. Elke G, Fuld MK, Halaweish AF, et al. Quantification of ventilation distribution in regional lung injury by electrical impedance tomography and xenon computed tomography. Physiol Meas 2013;34:1303-18.

44. Richard JC, Pouzot C, Gros A, et al. Electrical impedance tomography compared to positron emission tomography for the measurement of regional lung ventilation: an experimental study. Crit Care 2009;13:R82.

45. Shi C, Boehme S, Bentley AH, et al. Assessment of regional ventilation distribution: comparison of vibration response imaging (VRI) with electrical impedance tomography (EIT). PloS one 2014;9:e86638.

46. Shono A, Somhorst P, Gommers D. Electrical impedance tomography and trans-pulmonary pressure measurements in a patient with extreme respiratory drive. Respir Med Case Rep 2017;20:141-4. 